\title{
A LINK BETWEEN TWO ELLIPTIC QUANTUM GROUPS*
}

\author{
PAVEL ETINGOF ${ }^{\dagger}$ AND OLIVIER SCHIFFMANN ${ }^{\ddagger}$
}

\begin{abstract}
We consider the category $\mathcal{C}_{B}$ of meromorphic finite-dimensional representations of the quantum elliptic algebra $\mathcal{B}$ constructed via Belavin's R-matrix, and the category $\mathcal{C}_{F}$ of meromorphic finite-dimensional representations of Felder's elliptic quantum group $\mathcal{E}_{\tau, \frac{\gamma}{2}}\left(\mathfrak{g l}_{n}\right)$. For any fixed $c \in \mathbb{C}$, we use a version of the Vertex-IRF correspondence to construct two families of (generically) fully faithful functors $\mathcal{H}_{x}^{c}: \mathcal{C}_{B} \rightarrow \mathcal{D}_{B}$ and $\mathcal{F}_{x}^{c}: \mathcal{C}_{F} \rightarrow \mathcal{D}_{B}$ where $\mathcal{D}_{B}$ is a certain category of infinitedimensional representations of $\mathcal{B}$ by difference operators. We use this to construct an equivalence between the abelian subcategory of $\mathcal{C}_{B}$ generated by tensor products of vector representations and the abelian subcategory of $\mathcal{C}_{F}$ generated by tensor products of vector representations.
\end{abstract}

1. Categories of meromorphic representations. In this section, we recall the definitions of various categories of representations of quantum elliptic algebras.

Notations: Let us fix $\tau \in \mathbb{C}, \operatorname{Im}(\tau)>0, \gamma \in \mathbb{R} \backslash \mathbb{Q}$ and $n \geq 2$. Denote by $\left(v_{i}\right)_{i=1}^{n}$ the canonical basis of $\mathbb{C}^{n}$ and by $\left(E_{i j}\right)_{i, j=1}^{n}$ the canonical basis of $\operatorname{End}\left(\mathbb{C}^{n}\right)$, i.e $E_{i j} v_{k}=\delta_{j k} v_{i}$. Let $\mathfrak{h}=\left\{\sum_{i} \lambda_{i} E_{i i} \mid \sum_{i} \lambda_{i}=0\right\}$ be the space of diagonal traceless matrices. We have a natural identification $\mathfrak{h}^{*}=\left\{\sum_{i} \lambda_{i} E_{i i}^{*} \mid \sum_{i} \lambda_{i}=0\right\}$. In particular, the weight of $v_{i}$ is $\omega_{i}=E_{i i}^{*}-\frac{1}{n} \sum_{k} E_{k k}^{*}$.

Classical theta functions: The theta function $\theta_{\kappa, \kappa^{\prime}}(t ; \tau)$ with characteristics $\kappa, \kappa^{\prime} \in \mathbb{R}$ is defined by the formula

$$
\theta_{\kappa, \kappa^{\prime}}(t ; \tau)=\sum_{m \in \mathbb{Z}} e^{i \pi(m+\kappa)\left((m+\kappa) \tau+2\left(t+\kappa^{\prime}\right)\right)} .
$$

It is an entire function whose zeros are simple and form the (shifted) lattice $\left\{\frac{1}{2}-\kappa+\right.$ $\left.\left(\frac{1}{2}-\kappa^{\prime}\right) \tau\right\}+\mathbb{Z}+\tau \mathbb{Z}$.

Theta functions satisfy (and are characterized up to renormalization by) the following fundamental monodromy relations

$$
\begin{aligned}
& \theta_{\kappa, \kappa^{\prime}}(t+1 ; \tau)=e^{2 i \pi \kappa} \theta_{\kappa, \kappa^{\prime}}(t ; \tau), \\
& \theta_{\kappa, \kappa^{\prime}}(t+\tau ; \tau)=e^{-i \pi \tau-2 i \pi\left(t+\kappa^{\prime}\right)} \theta_{\kappa, \kappa^{\prime}}(t ; \tau) .
\end{aligned}
$$

Theta functions with different characteristics are related to each other by shifts of $t$ :

$$
\theta_{\kappa_{1}+\kappa_{2}, \kappa_{1}^{\prime}+\kappa_{2}^{\prime}}(t ; \tau)=e^{i \pi \kappa_{2}^{2} \tau+2 i \pi \kappa_{2}\left(t+\kappa_{1}^{\prime}+\kappa_{2}^{\prime}\right)} \theta_{\kappa_{1}, \kappa_{1}^{\prime}}\left(t+\kappa_{2} \tau+\kappa_{2}^{\prime} ; \tau\right)
$$

In particular, we set $\theta(t)=\theta_{\frac{1}{2}, \frac{1}{2}}(t ; \tau)$.

1.1. Meromorphic representations of the Belavin quantum elliptic algebra. Consider the two $n \times n$ matrices

$$
A=\left(\begin{array}{cccc}
1 & 0 & \ldots & 0 \\
0 & \xi & \ldots & 0 \\
\vdots & \vdots & \ddots & \vdots \\
0 & 0 & \ldots & \xi^{n-1}
\end{array}\right) \quad B=\left(\begin{array}{cccc}
0 & 1 & \ldots & 0 \\
\vdots & \vdots & \ddots & \vdots \\
0 & 0 & \vdots & 1 \\
1 & 0 & \ldots & 0
\end{array}\right)
$$

* Received March 20, 1998; accepted for publication June 16, 1998. The authors were supported by the NSF grant DMS-9700477. The second author would like to thank Harvard University Mathematics Department for its hospitality without which this work would not have been possible.

† Department of Mathematics, Harvard University, Cambridge MA 02138 USA (etingof@math. harvard.edu).

‡Department of Mathematics, ENS Paris, 45 rue d’ Ulm, 75005 Paris, FRANCE (schiffma@ sequoia.ens.fr). 
where $\xi=e^{2 i \pi / n}$. We have $A^{n}=B^{n}=I d, B A=\xi A B$, i.e $A, B$ generate the Heisenberg group. Belavin ([3]) introduced the matrix $R^{B}(z) \in \operatorname{End}\left(\mathbb{C}^{n}\right) \otimes \operatorname{End}\left(\mathbb{C}^{n}\right)$, uniquely determined by the following properties:

1. Unitarity: $R^{B}(z) R_{21}^{B}(-z)=1$,

2. $R^{B}(z)$ is meromorphic, with simple poles at $z=\gamma+\mathbb{Z}+\tau \mathbb{Z}$,

3. $R^{B}(0)=P: x \otimes y \mapsto y \otimes x$ for $x, y \in \mathbb{C}^{n}$ (permutation),

4. Lattice translation properties:

$$
\begin{aligned}
& R^{B}(z+1)=A_{1} R^{B}(z) A_{1}^{-1}=A_{2}^{-1} R^{B}(z) A_{2}, \\
& R^{B}(z+\tau)=e^{-2 i \pi \frac{n-1}{n} \gamma} B_{1} R^{B}(z) B_{1}^{-1}=e^{-2 i \pi \frac{n-1}{n} \gamma} B_{2}^{-1} R^{B}(z) B_{2} .
\end{aligned}
$$

In particular, $R^{B}(z)$ commutes with $A \otimes A$ and $B \otimes B$. The matrix $R^{B}(z)$ satisfies the quantum Yang-Baxter equation with spectral parameters:

$$
R_{12}^{B}(z-w) R_{13}^{B}(z) R_{23}^{B}(w)=R_{23}^{B}(w) R_{13}^{B}(z) R_{12}^{B}(z-w) .
$$

The category $\mathcal{C}_{B}$ : Following Faddeev, Reshetikhin, Takhtajan and SemenovTian-Shansky, one can define an algebra $\mathcal{B}$ from $R^{B}(z)$, using the RLL formalism-see [4], [12]. However, we will only need to consider a certain category of modules over this algebra, defined as follows.

Let $\mathcal{C}_{B}$ be the category whose objects are pairs $(V, L(z))$ where $V$ is a finite dimensional vector space and $L(z) \in \operatorname{End}\left(\mathbb{C}^{n}\right) \otimes \operatorname{End}(V)$ is an invertible meromorphic function (the L-operator) such that $L(z+n)=L(z)$ and $L(z+n \tau)=L(z)$, satisfying the following relation in the space $\operatorname{End}\left(\mathbb{C}^{n}\right) \otimes \operatorname{End}(V) \otimes \operatorname{End}(V)$ :

$$
R_{12}^{B}(z-w) L_{13}(z) L_{23}(w)=L_{23}(w) L_{13}(z) R_{12}^{B}(z-w)
$$

(as meromorphic functions of $z$ and $w$ ); morphisms $(V, L(z)) \rightarrow\left(V^{\prime}, L^{\prime}(z)\right)$ are linear maps $\varphi: V \rightarrow V^{\prime}$ such that $(1 \otimes \varphi) L(z)=L^{\prime}(z)(1 \otimes \varphi)$ in the space $\operatorname{Hom}\left(\mathbb{C}^{n} \otimes V, \mathbb{C}^{n} \otimes\right.$ $\left.V^{\prime}\right)$. The quantum Yang-Baxter relation for $R^{B}$ implies that $\left(\mathbb{C}^{n}, \chi(z) R^{B}(z-w)\right) \in$ $\mathcal{O} b\left(\mathcal{C}_{B}\right)$ for all $w \in \mathbb{C}$, where we set $\chi(z)=\frac{\theta\left(z-\left(1-\frac{1}{n}\right) \gamma\right)}{\theta(z)}$. This object is called the vector representation and will be denoted simply by $V_{B}(w)$.

The category $\mathcal{C}_{B}$ is naturally a tensor category with tensor product

$$
(V, L(z)) \otimes\left(V^{\prime}, L^{\prime}(z)\right)=\left(V \otimes V^{\prime}, L_{12}(z) L_{13}^{\prime}(z)\right)
$$

at the level of objects and with the usual tensor product at the level of morphisms.

There is a notion of a dual representation in the category $\mathcal{C}_{B}$ : the (right) dual of $(V, L(z))$ is $\left(V^{*}, L^{*}(z)\right)$ where $L^{*}(z)=L^{-1}(z)^{t_{2}}$ (first apply inversion, then apply the transposition in the second component $\left.t_{2}\right)$. If $V, W \in \mathcal{O} b\left(\mathcal{C}_{B}\right)$ and $\varphi \in \operatorname{Hom}_{\mathcal{C}_{B}}(V, W)$ then $\varphi^{t} \in \operatorname{Hom}_{\mathcal{C}_{B}}\left(W^{*}, V^{*}\right)$, and the functor $\mathcal{C}_{B} \rightarrow \mathcal{C}_{B}, V \mapsto V^{*}$ is a contravariant equivalence of categories. Moreover, for $V, W, Z \in \mathcal{O} b\left(\mathcal{C}_{B}\right)$, we have canonical isomorphisms $(V \otimes W)^{*} \simeq\left(W^{*} \otimes V^{*}\right)$ and $\operatorname{Hom}_{\mathcal{C}_{B}}(V \otimes W, Z) \simeq \operatorname{Hom}_{\mathcal{C}_{B}}\left(V, Z \otimes W^{*}\right)$.

We will also need an extended category $\mathcal{C}_{B}^{x}$ defined as follows: objects of $\mathcal{C}_{B}^{x}$ are objects of $\mathcal{C}_{B}$ but we set

$$
\operatorname{Hom}_{\mathcal{C}_{B}^{x}}\left(V, V^{\prime}\right)=\operatorname{Hom}_{\mathcal{C}_{B}}\left(V, V^{\prime}\right) \otimes M_{\mathbb{C}}
$$

where $M_{\mathbb{C}}$ is the field of meromorphic functions of a complex variable $x$. In other words, morphisms in $\mathcal{C}_{B}^{x}$ are meromorphic 1-parameter families of morphisms in $\mathcal{C}_{B}$. 
The category $\mathcal{D}_{B}:$ We now define a difference-operator variant of the categories $\mathcal{C}_{B}, \mathcal{C}_{B}^{x}$. Let us denote by $M_{\mathfrak{h}^{*}}$ the field of $\left(n \omega_{i}\right)$-periodic meromorphic functions $\mathfrak{h}^{*} \rightarrow \mathbb{C}$ and by $D_{\mathfrak{h}^{*}}$ the $\mathbb{C}$-algebra generated by $M_{\mathfrak{h}^{*}}$ and shift operators $T_{\mu}: M_{\mathfrak{h}^{*}} \rightarrow$ $M_{\mathfrak{h}^{*}}, f(\lambda) \mapsto f(\lambda+\mu)$ for $\mu \in \mathfrak{h}^{*}$. If $V$ is a finite-dimensional vector space, we set $V_{\mathfrak{h}^{*}}=M_{\mathfrak{h}^{*}} \otimes_{\mathfrak{C}} V$, and $D(V)=D_{\mathfrak{h}^{*}} \otimes_{\mathbb{C}} \operatorname{End}(V)$. Let $\mathcal{D}_{B}$ be the category whose objects are pairs $(V, L(z))$ where $V$ is a finite-dimensional $\mathbb{C}$-vector space and $L(z) \in$ $\operatorname{End}\left(\mathbb{C}^{n}\right) \otimes D(V)$ is an invertible operator with meromorphic coefficients satisfying (4) in $\operatorname{End}\left(\mathbb{C}^{n}\right) \otimes D(V) \otimes D(V)$; morphisms $(V, L(z)) \rightarrow\left(V^{\prime}, L^{\prime}(z)\right)$ are $\left(n \omega_{i}\right)$-periodic meromorphic functions $\varphi: \mathfrak{h}^{*} \rightarrow \operatorname{Hom}\left(V, V^{\prime}\right)$ such that $(1 \otimes \varphi) L(z)=L(z)(1 \otimes \varphi)$ in $\operatorname{Hom}_{\mathbb{C}}\left(\mathbb{C}^{n} \otimes V_{\mathfrak{h}^{*}}, \mathbb{C}^{n} \otimes V_{\mathfrak{h}^{*}}^{\prime}\right)$ (i.e morphisms are $M_{\mathfrak{h}^{*}}$-linear).

The category $\mathcal{D}_{B}$ is a right-module category over $\mathcal{C}_{B}$, i.e we have a (bi)functor $\otimes$ : $\mathcal{D}_{B} \times \mathcal{C}_{B} \rightarrow \mathcal{D}_{B}$ defined by (5), and for any $V, W \in \mathcal{O} b\left(\mathcal{D}_{B}\right), Z \in \mathcal{O} b\left(\mathcal{C}_{B}\right)$, we have a canonical isomorphism $\operatorname{Hom}_{\mathcal{D}_{B}}(V \otimes Z, W) \simeq \operatorname{Hom}_{\mathcal{D}_{B}}\left(V, W \otimes Z^{*}\right)$. The category $\mathcal{D}_{B}^{x}$ is defined in an analogous way: objects are pairs $(V, L(z, x))$ as in $\mathcal{D}_{B}$ but the L-operator is now a meromorphic function of $z$ and $x$, and morphisms $(V, L(z, x)) \rightarrow\left(V^{\prime}, L^{\prime}(z, x)\right)$ are meromorphic maps $\varphi(\lambda, x): \mathfrak{h}^{*} \times \mathbb{C} \rightarrow \operatorname{Hom}_{\mathbb{C}}\left(V, V^{\prime}\right)$ satisfying $(1 \otimes \varphi) L(z, x)=$ $L(z, x)(1 \otimes \varphi)$.

\subsection{Meromorphic representations of the elliptic quantum group} $\mathcal{E}_{\tau, \gamma / 2}\left(\mathfrak{g l}_{n}\right)$.

Felder's dynamical R-matrix: let us consider the functions of two complex variables

$$
\alpha(z, l)=\frac{\theta(l+\gamma) \theta(z)}{\theta(l) \theta(z-\gamma)}, \quad \beta(z, l)=\frac{\theta(z-l) \theta(\gamma)}{\theta(l) \theta(z-\gamma)} .
$$

As functions of $z, \alpha$ and $\beta$ have simple poles at $z=\gamma+\mathbb{Z}+\tau \mathbb{Z}$ and satisfy

$$
\begin{array}{ll}
\alpha(z+1, l)=\alpha(z, l), & \alpha(z+\tau, l)=e^{-2 i \pi \gamma} \alpha(z, l), \\
\beta(z+1, l)=\beta(z, l), & \beta(z+\tau, l)=e^{-2 i \pi(\gamma-l)} \beta(z, l) .
\end{array}
$$

Felder introduced in [5] the matrix $R^{F}(z, \lambda): \mathbb{C} \times \mathfrak{h}^{*} \rightarrow \operatorname{End}\left(\mathbb{C}^{n}\right) \otimes \operatorname{End}\left(\mathbb{C}^{n}\right)$ :

$$
R^{F}(z, \lambda)=\sum_{i} E_{i i} \otimes E_{i i}+\sum_{i \neq j} \alpha\left(z, \lambda_{i}-\lambda_{j}\right) E_{i i} \otimes E_{j j}+\sum_{i \neq j} \beta\left(z, \lambda_{i}-\lambda_{j}\right) E_{j i} \otimes E_{i j}
$$

where $\lambda=\sum_{i} \lambda_{i} E_{i i}^{*} \in \mathfrak{h}^{*}$.

This matrix is a solution of the quantum dynamical Yang-Baxter equation with spectral parameters

$$
\begin{aligned}
& R_{12}^{F}\left(z-w, \lambda-\gamma h_{3}\right) R_{13}^{F}(z, \lambda) R_{23}^{F}\left(w, \lambda-\gamma h_{1}\right) \\
& =R_{23}^{F}(w, \lambda) R_{13}^{F}\left(z, \lambda-\gamma h_{2}\right) R_{12}^{F}(z-w, \lambda)
\end{aligned}
$$

where we have used the following convention: if $V_{i}$ are diagonalizable $\mathfrak{h}$-modules with weight decomposition $V_{i}=\bigoplus_{\mu} V_{i}^{\mu}$ and $a(\lambda) \in \operatorname{End}\left(\bigotimes_{i} V_{i}\right)$ then

$$
a\left(\lambda-\gamma h_{l}\right)_{\mid \otimes_{i} V_{i}^{\mu_{i}}}=a\left(\lambda-\gamma \mu_{l}\right) \text {. }
$$

As usual, indices indicate the components of the tensor product on which the operators act.

In addition, $R^{F}(z, \lambda)$ satisfies the following two conditions:

1. Unitarity: $R_{12}^{F}(z, \lambda) R_{21}^{F}(-z, \lambda)=I d$, 
2. Weight zero: $\forall h \in \mathfrak{h},\left[h_{1}+h_{2}, R^{F}(z, \lambda)\right]=0$.

The category $\mathcal{C}_{F}$ : It is possible to use $R^{F}(z, \lambda)$ to define an algebra by the RLL-formalism (see [5]): the elliptic quantum group $\mathcal{E}_{\tau, \gamma / 2}\left(\mathfrak{g l}_{n}(\mathbb{C})\right)$. However, we will only need the following category of its representations $\mathcal{C}_{F}$, introduced by Felder in [5] and studied by Felder and Varchenko in [6]: objects are pairs $(V, L(z, \lambda))$ where $V$ is a finite-dimensional diagonalizable $\mathfrak{h}$-module and $L(z, \lambda): \mathbb{C} \times \mathfrak{h}^{*} \rightarrow \operatorname{End}\left(\mathbb{C}^{n}\right) \otimes \operatorname{End}(V)$ is an invertible meromorphic function which is $\left(n \omega_{i}\right)$-periodic in $\lambda$ and which satisfies the following two conditions:

$$
\begin{gathered}
{\left[h_{1}+h_{2}, L(z, \lambda)\right]=0} \\
R_{12}^{F}\left(z-w, \lambda-\gamma h_{3}\right) L_{13}(z, \lambda) L_{23}\left(w, \lambda-\gamma h_{1}\right) \\
=L_{23}(w, \lambda) L_{13}\left(z, \lambda-\gamma h_{2}\right) R_{12}^{F}(z-w, \lambda) .
\end{gathered}
$$

Morphisms $(V, L(z, \lambda)) \rightarrow\left(V^{\prime}, L^{\prime}(z, \lambda)\right)$ are $\left(n \omega_{i}\right)$-periodic meromorphic weight zero maps $\varphi(\lambda): V \rightarrow V^{\prime}$ such that $L^{\prime}(z, \lambda)\left(1 \otimes \varphi\left(\lambda-\gamma h_{1}\right)\right)=(1 \otimes \varphi(\lambda)) L(z, \lambda)$. The quantum dynamical Yang-Baxter relation for $R^{F}(z, \lambda)$ implies that we have $\left(\mathbb{C}^{n}, R^{F}(z-w, \lambda)\right) \in \mathcal{O} b\left(\mathcal{C}_{F}\right)$ for all $w \in \mathbb{C}$. This is the vector representation and it will be denoted by $V_{F}(w)$.

The category $\mathcal{C}_{F}$ is naturally equipped with a tensor structure: it is defined on objects by

$$
(V, L(z, \lambda)) \otimes\left(V^{\prime}, L^{\prime}(z, \lambda)\right)=\left(V \otimes V^{\prime}, L_{12}\left(z, \lambda-\gamma h_{3}\right) L_{13}^{\prime}(z, \lambda)\right),
$$

and if $\varphi \in \operatorname{Hom}_{\mathcal{C}_{F}}(V, W), \varphi^{\prime} \in \operatorname{Hom}_{\mathcal{C}_{F}}\left(V^{\prime}, W^{\prime}\right)$ then

$$
\left(\varphi \otimes \varphi^{\prime}\right)(\lambda)=\varphi\left(\lambda-\gamma h_{2}\right) \otimes \varphi^{\prime}(\lambda) \in \operatorname{Hom}_{\mathcal{C}_{F}}\left(V \otimes V^{\prime}, W \otimes W^{\prime}\right) .
$$

There is a notion of a dual representation in the category $\mathcal{C}_{F}$ : the (right) dual of $(V, L(z, \lambda))$ is $\left(V^{*}, L^{*}(z, \lambda)\right)$ where $L^{*}(z, \lambda)=L^{-1}\left(z, \lambda+\gamma h_{2}\right)^{t_{2}}$ (apply inversion, shifting and then apply the transposition in the second component $t_{2}$ ). If $V, W \in$ $\mathcal{O} b\left(\mathcal{C}_{F}\right)$ and $\varphi(\lambda) \in \operatorname{Hom}_{\mathcal{C}_{F}}(V, W)$ then $\varphi^{*}(\lambda):=\varphi\left(\lambda+\gamma h_{1}\right)^{t} \in \operatorname{Hom}_{\mathcal{C}_{F}}\left(W^{*}, V^{*}\right)$, and the functor $\mathcal{C}_{F} \rightarrow \mathcal{C}_{F}, V \mapsto V^{*}$ is a contravariant equivalence of categories. Moreover, for any $V, W \in \mathcal{O} b\left(\mathcal{C}_{F}\right)$, there is a canonical isomorphism $(V \otimes W)^{*} \simeq\left(W^{*} \otimes V^{*}\right)$.

The extended category $\mathcal{C}_{F}^{x}$ is defined by $\mathcal{O} b\left(\mathcal{C}_{F}^{x}\right)=\mathcal{O} b\left(\mathcal{C}_{F}\right)$ and

$$
\operatorname{Hom}_{\mathcal{C}_{F}^{x}}\left(V, V^{\prime}\right)=\operatorname{Hom}_{\mathcal{C}_{F}}\left(V, V^{\prime}\right) \otimes M_{\mathbb{C}}
$$

i.e morphisms in $\mathcal{C}_{F}^{x}$ are meromorphic 1-parameter families of morphisms in $\mathcal{C}_{F}$.

2. The functor $\mathcal{F}_{x}^{c}: \mathcal{C}_{F} \rightarrow \mathcal{D}_{B}$. In this section, we define a family of functors from meromorphic (finite-dimensional) representations of $\mathcal{E}_{\tau, \frac{\gamma}{2}}\left(\mathfrak{g l}_{n}(\mathbb{C})\right.$ ) to infinitedimensional representations of the quantum elliptic algebra $\mathcal{B}$.

2.1. Twists by difference operators. For any finite-dimensional diagonalizable $\mathfrak{h}$-module $V$, let $e^{\gamma D} \in \operatorname{End}\left(V_{\mathfrak{h}^{*}}\right)$ denote the shift operator: $e^{\gamma D} \cdot \sum_{\mu} f_{\mu}(\lambda) v_{\mu}=$ $\sum_{\mu} f(\lambda+\gamma \mu) v_{\mu}, v_{\mu} \in V_{\mu}$. Now let $(V, L(z, \lambda)) \in \mathcal{C}_{F}$, and let $S(z, \lambda), S^{\prime}(z, \lambda)$ : $\mathbb{C} \times \mathfrak{h}^{*} \rightarrow \operatorname{End}\left(\mathbb{C}^{n}\right)$ be meromorphic and nondegenerate. Define the difference-twist of $(V, L(z, \lambda))$ to be the pair $\left(V, L^{S, S^{\prime}}(z)\right)$ where

$$
L^{S, S^{\prime}}(z)=S_{1}\left(z, \lambda-\gamma h_{2}\right) L(z, \lambda) e^{-\gamma D_{1}} S_{1}^{\prime}(z, \lambda)^{-1} \in \operatorname{End}\left(\mathbb{C}^{n}\right) \otimes D(V) .
$$


This is a difference operator acting on $\mathbb{C}^{n} \otimes V_{\mathfrak{h}^{*}}$.

LEMMA 1. The difference operator $L^{S}(z, \lambda)$ satisfies the following relation in $\operatorname{End}\left(\mathbb{C}^{n}\right) \otimes D(V) \otimes D(V):$

$$
T_{12}\left(z, w, \lambda-\gamma h_{3}\right) L_{13}^{S, S^{\prime}}(z) L_{23}^{S, S^{\prime}}(w)=L_{23}^{S, S^{\prime}}(w) L_{13}^{S, S^{\prime}}(z) T_{12}^{\prime}(z, w, \lambda)
$$

where

$$
\begin{aligned}
T(z, w, \lambda) & =S_{2}(w, \lambda) S_{1}\left(z, \lambda-\gamma h_{2}\right) R_{12}^{F}(z-w, \lambda) S_{2}\left(w, \lambda-\gamma h_{1}\right)^{-1} S_{1}(z, \lambda)^{-1}, \\
T^{\prime}(z, w, \lambda) & =S_{1}^{\prime}(z, \lambda) S_{2}^{\prime}\left(w, \lambda+\gamma h_{1}\right) R_{12}^{F}(z-w, \lambda) S_{1}^{\prime}\left(z, \lambda+\gamma h_{1}\right)^{-1} S_{2}^{\prime}(w, \lambda)^{-1} .
\end{aligned}
$$

Proof. The proof is straightforward, using relation (6) for $L(z, \lambda)$ and the weight zero property of $R^{F}(u, \lambda)$ and $L(u, \lambda)$.

2.2. The Vertex-IRF transform. Let $\phi_{l}(u)=e^{2 i \pi\left(\frac{l^{2} \tau}{n}+\frac{l u}{n}\right)} \theta_{0,0}(u+l \tau ; n \tau)$ for $l=1, \ldots n$. Then the vector $\Phi(u)=\left(\phi_{1}(u), \ldots, \phi_{n}(u)\right)$ is, up to renormalization, the unique holomorphic vector in $\mathbb{C}^{n}$ satisfying the following monodromy relations:

$$
\begin{aligned}
& \Phi(u+1)=A \Phi(u), \\
& \Phi(u+\tau)=e^{-i \pi \frac{\tau}{n}-2 i \pi \frac{u}{n}} B \Phi(u) .
\end{aligned}
$$

Now let $S(z, \lambda): \mathbb{C} \times \mathfrak{h}^{*} \rightarrow \operatorname{End}\left(\mathbb{C}^{n}\right)$ be the matrix whose columns are $\left(\Phi_{1}(z, \lambda), \ldots\right.$, $\left.\Phi_{n}(z, \lambda)\right)$ where $\Phi_{j}(z, \lambda)=\Phi\left(z-n \lambda_{j}\right)$. Using (10)-(11), it is easy to see that we have $\operatorname{det}(S(z, \lambda))=\operatorname{Const}(\lambda) \theta(z)$ where $\operatorname{Const}(\lambda) \neq 0$ and hence that $S(z, \lambda)$ is invertible for $z \neq 0$ and generic $\lambda$.

LEMMA 2. We have

$$
\begin{aligned}
& R^{B}(z-w) S_{1}(z, \lambda) S_{2}\left(w, \lambda-\gamma h_{1}\right)=S_{2}(w, \lambda) S_{1}\left(z, \lambda-\gamma h_{2}\right) R^{F}(z-w, \lambda), \\
& R^{B}(z-w) S_{2}(w, \lambda) S_{1}\left(z, \lambda+\gamma h_{2}\right)=S_{1}(z, \lambda) S_{2}\left(w, \lambda+\gamma h_{1}\right) R^{F}(z-w, \lambda) .
\end{aligned}
$$

Proof. The first relation is equivalent to the following identities for $i, j=1, \ldots n$ :

$$
\begin{aligned}
R^{B}(z-w) \Phi_{i}(z, \lambda) \otimes \Phi_{i}\left(w, \lambda-\gamma \omega_{i}\right) & =\Phi_{i}\left(z, \lambda-\gamma \omega_{i}\right) \otimes \Phi_{i}(w, \lambda) \\
R^{B}(z-w) \Phi_{i}(z, \lambda) \otimes \Phi_{j}\left(w, \lambda-\gamma \omega_{i}\right) & =\alpha\left(z-w, \lambda_{i}-\lambda_{j}\right) \Phi_{i}\left(z, \lambda-\gamma \omega_{j}\right) \otimes \Phi_{j}(w, \lambda) \\
& +\beta\left(z-w, \lambda_{i}-\lambda_{j}\right) \Phi_{j}\left(z, \lambda-\gamma \omega_{i}\right) \otimes \Phi_{i}(w, \lambda) .
\end{aligned}
$$

These identities are proved by comparing poles and transformation properties under lattice translations as functions of $z$ and $w$, and using the uniqueness of $\Phi$. The second relation of the lemma is proved in the same way. These identities are essentially the Vertex/Interaction-Round-a-Face transform of statistical mechanics (see [9],[11] and [7] for the case $n=2$ ).

The Vertex-IRF transform first appeared in the work of Baxter [1] and was subsequently generalized to the Belavin R-matrix by Jimbo, Miwa and Okado in [10].

2.3. Construction of the functor $\mathcal{F}_{x}^{c}: \mathcal{C}_{F} \rightarrow \mathcal{D}_{B}$. Let us fix some $c \in \mathbb{C}$. We now define the family of functors $\mathcal{F}_{x}^{c}: \mathcal{C}_{F} \rightarrow \mathcal{C}_{B}$ indexed by $x \in \mathbb{C}$ : for $(V, L(z, \lambda)) \in$ $\mathcal{C}_{F}$, set $\mathcal{F}_{x}^{c}((V, L(z, \lambda)))=\left(V, L^{S_{x}, S_{x+c}}(z)\right)$ with $S_{u}(z, \lambda)=S(z-u, \lambda)$ as above and let $\mathcal{F}_{x}^{c}$ be trivial at the level of morphisms.

Proposition 1. $\mathcal{F}_{x}^{c}: \mathcal{C}_{F} \rightarrow \mathcal{D}_{B}$ is a functor. 
Proof. It follows from Lemma 2 that $\left(V, L^{S_{x}, S_{x+c}}(z)\right) \in \mathcal{O} b\left(\mathcal{D}_{B}\right)$. Furthermore, if $\varphi(\lambda) \in \operatorname{Hom}_{\mathcal{C}_{F}}\left((V, L(z, \lambda)),\left(V^{\prime}, L^{\prime}(z, \lambda)\right)\right)$ then by definition we have $L^{\prime}(z, \lambda)(1 \otimes$ $\left.\varphi\left(\lambda-\gamma h_{1}\right)\right)=(1 \otimes \varphi(\lambda)) L(z, \lambda)$, so that

$$
\begin{aligned}
S_{1}(z-x, \lambda & \left.-\gamma h_{2}\right) L^{\prime}(z, \lambda) e^{-\gamma D_{1}} S_{1}(z-x-c, \lambda)^{-1}(1 \otimes \varphi(\lambda)) \\
& =S_{1}\left(z-x, \lambda-\gamma h_{2}\right) L^{\prime}(z, \lambda)\left(1 \otimes \varphi\left(\lambda-\gamma h_{1}\right)\right) e^{-\gamma D_{1}} S_{1}(z-x-c, \lambda)^{-1} \\
& =S_{1}\left(z-x, \lambda-\gamma h_{2}\right)(1 \otimes \varphi(\lambda)) L^{\prime}(z, \lambda) e^{-\gamma D_{1}} S_{1}(z-x-c, \lambda)^{-1} \\
& =(1 \otimes \varphi(\lambda)) S_{1}\left(z-x, \lambda-\gamma h_{2}\right) L^{\prime}(z, \lambda) e^{-\gamma D_{1}} S_{1}(z-x-c, \lambda)^{-1}
\end{aligned}
$$

since $\varphi(\lambda)$ is of weight zero. Thus $\mathcal{F}_{x}^{c}(\varphi(\lambda))$ is an intertwiner in the category $\mathcal{D}_{B}$.

We can also think of the family of functors $\mathcal{F}_{x}^{c}$ as a single functor $\mathcal{F}^{c}: \mathcal{C}_{F}^{x} \rightarrow \mathcal{D}_{B}^{x}$.

REMARK. We can think of the difference-twist and the relations in Lemma 2 as a dynamical analogue of the notion of equivalence of R-matrices due to Drinfeld and Belavin-see [2].

3. The image of the trivial representation and the functor $\mathcal{H}_{x}^{c}: \mathcal{C}_{B} \rightarrow$ $\mathcal{D}_{B}$. Applying the functor $\mathcal{F}_{x}^{c}$ to the trivial representation $(\mathbb{C}, \mathrm{Id}) \in \mathcal{O} b\left(\mathcal{C}_{F}\right)$ yields

$$
\mathcal{F}_{x}^{c}((\mathbb{C}, \mathrm{Id}))=\left(\mathbb{C}, S(z-x, \lambda) e^{-\gamma D_{1}} S(z-x-c, \lambda)^{-1}\right) .
$$

We will denote this object by $I_{x}^{c}$. For instance, when $n=2$, we obtain a representation of the Belavin quantum elliptic algebra as difference operators acting on the space of periodic meromorphic functions in one variable $\lambda$, i.e given by an L-operator

$$
L(z)=\left(\begin{array}{ll}
a(z) & b(z) \\
c(z) & d(z)
\end{array}\right)
$$

where $a(z), b(z), c(z), d(z)$ are operators of the form $f(z) T_{-\gamma}+g(z)$ where $T_{-\gamma}$ is the shift by $-\gamma$.

Such representations of $\mathcal{B}$ by difference operators already appeared in the work of Krichever, Zabrodin ([11]) (for $n=2$ ) and Hasegawa ([8],[9])(for the general case), where they were also derived by some Vertex-IRF correspondence.

Definition. Let $c \in \mathbb{C}$ and let $\mathcal{H}_{x}^{c}: \mathcal{C}_{B} \rightarrow \mathcal{D}_{B}$ be the functor defined by the assignment $V \rightarrow I_{x}^{c} \otimes V$ and which is trivial at the level of morphisms. The family of functors $\mathcal{H}_{x}^{c}$ gives rise to a functor $\mathcal{H}^{c}: \mathcal{C}_{B}^{x} \rightarrow \mathcal{D}_{B}^{x}$.

4. Full faithfulness of the functor $\mathcal{H}_{x}^{c}: \mathcal{C}_{B} \rightarrow \mathcal{D}_{B}$. In this section, we prove the following result

Proposition 2. Let $V, V^{\prime} \in \mathcal{O} b\left(\mathcal{C}_{B}\right)$. Then for all but finitely many values of $x \bmod \mathbb{Z}+\mathbb{Z} \tau$, the map

$$
\mathcal{H}_{x}^{c}: \operatorname{Hom}_{\mathcal{C}_{B}}\left(V, V^{\prime}\right) \stackrel{\sim}{\rightarrow} \operatorname{Hom}_{\mathcal{D}_{B}}\left(\mathcal{H}_{x}^{c}(V), \mathcal{H}_{x}^{c}\left(V^{\prime}\right)\right)
$$

is an isomorphism.

Proof. Since $\operatorname{Hom}_{\mathcal{C}_{B}}\left(V, V^{\prime}\right) \simeq \operatorname{Hom}_{\mathcal{C}_{B}}\left(\mathbb{C}, V^{\prime} \otimes V^{*}\right), \operatorname{Hom}_{\mathcal{D}_{B}}\left(I_{x}^{c} \otimes V, I_{x}^{c} \otimes V^{\prime}\right) \simeq$ $\operatorname{Hom}_{\mathcal{D}_{B}}\left(I_{x}^{c}, I_{x}^{c} \otimes V^{\prime} \otimes V^{*}\right)$, it is enough to show that the map $\mathcal{H}_{x}^{c}: \operatorname{Hom}_{\mathcal{C}_{B}}(\mathbb{C}, W) \rightarrow$ $\operatorname{Hom}_{\mathcal{D}_{B}}\left(I_{x}^{c}, I_{x}^{c} \otimes W\right)$ is an isomorphism for all $W \in \mathrm{Ob}\left(\mathcal{C}_{B}\right)$. Since $\mathcal{H}_{x}^{c}$ is trivial at the level of morphisms, this map is injective. Now let $W \in \mathcal{O} b\left(\mathcal{C}_{B}\right)$ and let $\varphi(\lambda) \in$ $\operatorname{Hom}_{\mathcal{D}_{B}}\left(I_{x}^{c}, I_{x}^{c} \otimes W\right)$, that is, $\varphi(\lambda)$ is a $\left(n \omega_{i}\right)$-periodic meromorphic function $\mathfrak{h}^{*} \rightarrow W$ satisfying the equation

$$
\begin{aligned}
\varphi_{2}(\lambda) S_{1}(z-x, \lambda) e^{-\gamma D_{1}} S_{1}(z-x-c, \lambda)^{-1} \\
\quad=S_{1}(z-x, \lambda) e^{-\gamma D_{1}} S_{1}(z-x-c, \lambda)^{-1} L_{12}(z) \varphi_{2}(\lambda)
\end{aligned}
$$


where $L(z)$ is the L-operator of $W$. This is equivalent to

$$
L_{12}(z) \varphi_{2}(\lambda)=S_{1}(z-x-c, \lambda) \varphi_{2}\left(\lambda+\gamma h_{1}\right) S_{1}(z-x-c, \lambda)^{-1}
$$

Now $L(z)$ is an elliptic function (of periods $n$ and $n \tau$ ) so it is either constant or it has a pole. Restricting $W$ to the subrepresentation $\operatorname{Span}\left(\varphi(\lambda), \lambda \in \mathfrak{h}^{*}\right)$, we see that the latter case is impossible for generic $x$ as the RHS of (12) has a pole at $z=x+c$ only; hence $L(z)$ is constant. Furthermore, from (12) we see that the matrix

$$
M(\lambda)=S_{1}(z-x-c, \lambda)^{-1} L_{12} S_{1}(z-x-c, \lambda)
$$

is independent of $z$. In particular, setting $z \mapsto z+1$ and using the transformation properties (10) of $S(z, \lambda)$, we obtain $\left[A_{1}, L_{12}\right]=0$. This implies that $L=\sum_{i} E_{i i} \otimes D_{i}$ for some $D_{i} \in \operatorname{End}(W)$.

LEMma 3. Let $U$ be a finite dimensional vector space, let $T \in \operatorname{End}\left(\mathbb{C}^{n}\right) \otimes \operatorname{End}(U)$ be an invertible solution of the equation

$$
R_{12}^{B}(z) T_{13} T_{23}=T_{23} T_{13} R_{12}^{B}(z)
$$

such that $T=\sum_{i} E_{i i} \otimes D_{i}$ for some $D_{i} \in \operatorname{End}(U)$. Then $\left[D_{i}, D_{j}\right]=0$ for all $i, j$ and there exists $X \in \operatorname{End}(U)$ such that $X^{n}=1$ and $D_{i+1}=X D_{i}$ for all $i=1, \ldots n$.

Proof. Let us write $R^{B}(z)=\sum_{p, q, r, s} R_{p, q, r, s}(z) E_{p q} \otimes E_{r s}$. Then equation (13) is equivalent to $R_{p, q, r, s}(z) D_{p} \times D_{q}=R_{p, q, r, s}(z) D_{s} D_{r}$ for all $p, q, r, s$. But it follows from the general formula for $R^{B}(z)$ that $R_{p, q, r, s}(z) \neq 0$ if and only if $p+q \equiv r+s(\bmod n)$. Thus we have $\left[D_{i}, D_{j}\right]=0$ for all $i, j$ and $X:=D_{i} D_{i+1}^{-1}$ is independent of $i$, and satisfies $X^{n}=1$.

By the above lemma, there exists $X \in \operatorname{End}(W)$ such that $X^{n}=1$ and $D_{i+1}=$ $X D_{i}$. Suppose that $X \neq 1$ and choose $e \in W$ such that $X(e)=\xi^{k} e$ with $\xi^{k} \neq 1$. Now we apply the transformation $z \mapsto z+\tau$ to the matrix $M(\lambda)$. Noting that, by (11), $S(z-x-c+\tau, \lambda)=e^{-i \pi \tau / 2-2 i \pi(z-x-c) / n} B S(z-x-c, \lambda) F(\lambda)$ where $F(\lambda)=$ $\operatorname{diag}\left(e^{-2 i \pi \lambda}, \ldots e^{-2 i \pi \lambda_{n}}\right)$, we obtain the equality

$$
\begin{aligned}
F(\lambda)^{-1} S_{1}(z-x-c, \lambda)^{-1} B_{1}^{-1} L_{12} B_{1} & S_{1}(z-x-c, \lambda) F(\lambda) \\
& =S_{1}(z-x-c, \lambda)^{-1} L_{12} S_{1}(z-x-c, \lambda) .
\end{aligned}
$$

Applying this to the vector $e$ yields $\operatorname{Ad} F(\lambda)(M(\lambda))(e)=\xi^{-k} M(\lambda)(e)$. This is possible for all $\lambda$ only if $k \equiv 0(\bmod n)$. Hence $X=1$ and $(12)$ reduces to the equation $D \varphi_{2}(\lambda)=\varphi_{2}\left(\lambda+\gamma h_{1}\right)$. In particular $\varphi(\lambda)$ is $\gamma\left(\omega_{i}-\omega_{j}\right)$-periodic. But by our assumption, $\varphi(\lambda)$ is $\left(n \omega_{i}\right)$-periodic and $\gamma$ is real and irrational. Therefore $\varphi(\lambda)$ is constant and it is a morphism in the category $\mathcal{C}_{B}$.

Now, considering $x$ as a parameter, we obtain:

Corollary 1. The functor $\mathcal{H}^{c}: \mathcal{C}_{B}^{x} \rightarrow \mathcal{D}_{B}^{x}$ is fully faithful.

REMARK. Equation (12) shows that $\operatorname{Hom}_{\mathcal{D}_{B}}\left(I_{x}^{c}, I_{x}^{c} \otimes V\right)=\operatorname{Hom}_{\mathcal{D}_{B}}\left(V^{*}, I_{x+c}^{0}\right)$. Thus the above proposition states that for any finite-dimensional representation $V \in$ $\mathcal{O} b\left(\mathcal{C}_{F}\right)$ and for all but finitely many $x \bmod \mathbb{Z}+\tau \mathbb{Z}$, we have $\operatorname{Hom}_{\mathcal{D}_{B}}\left(V^{*}, I_{x}^{0}\right)=$ $\operatorname{Hom}_{\mathcal{C}_{B}}\left(V^{*}, \mathbb{C}\right)$, where the isomorphism is induced by the embedding $\mathbb{C} \subset I_{x}^{0}$ (constant functions). However, for finitely many values of $x \bmod \mathbb{Z}+\tau \mathbb{Z}$, this may not be true: see [11] and [9] where some finite-dimensional subrepresentations of $I_{x}^{0}$ are considered. 
5. Full faithfullness of the functor $\mathcal{F}_{x}^{c}: \mathcal{C}_{F} \rightarrow \mathcal{D}_{B}$. In this section, we prove the following result:

Proposition 3. The functor $\mathcal{F}_{x}^{c}: \mathcal{C}_{F} \rightarrow \mathcal{D}_{B}$ is fully faithful.

Proof. We have to show that for any two objects $V, V^{\prime}$ in $\mathcal{C}_{F}$ there is an isomorphism $\mathcal{F}_{x}^{c}: \operatorname{Hom}_{\mathcal{C}_{F}}\left(V, V^{\prime}\right) \rightarrow \operatorname{Hom}_{\mathcal{D}_{B}}\left(\mathcal{F}_{x}^{c}(V), \mathcal{F}_{x}^{c}\left(V^{\prime}\right)\right)$. Since $\mathcal{F}_{x}^{c}$ is trivial at the level of morphisms, this map is injective. Now let $V, W \in \mathcal{O} b\left(\mathcal{C}_{F}\right)$ and let $\varphi(\lambda) \in \operatorname{Hom}_{\mathcal{D}_{B}}\left(\mathcal{F}_{x}^{c}(V), \mathcal{F}_{x}^{c}(W)\right)$. By definition, $\varphi(\lambda): V \rightarrow W$ satisfies the relation

$$
\begin{aligned}
\varphi_{2}(\lambda) S_{1}\left(z-x, \lambda-\gamma h_{2}\right) L_{12}^{V}(z, \lambda) e^{-\gamma D_{1}} S_{1}(z-x-c, \lambda)^{-1} \\
\quad=S_{1}\left(z-x, \lambda-\gamma h_{2}\right) L_{12}^{W}(z, \lambda) e^{-\gamma D_{1}} S_{1}(z-x-c, \lambda)^{-1} \varphi_{2}(\lambda)
\end{aligned}
$$

where $L^{V}(z, \lambda)$ (resp. $\left.L^{W}(z, \lambda)\right)$ is the L-operator of $V$ (resp. $W$ ). This is equivalent to

$$
\varphi_{2}(\lambda) S_{1}\left(z-x, \lambda-\gamma h_{2}\right) L_{12}^{V}(z, \lambda)=S_{1}\left(z-x, \lambda-\gamma h_{2}\right) L_{12}^{W}(z, \lambda) \varphi_{2}\left(\lambda-\gamma h_{1}\right)
$$

Introduce the following notations: write $W=\bigoplus_{\xi} W_{\xi}, V=\bigoplus_{\mu} V_{\mu}, \varphi(\lambda)=$ $\sum_{\nu} \varphi_{\nu}(\lambda)$ for the weight decompositions (so that $\varphi_{\nu}: V_{\xi} \rightarrow W_{\xi+\nu}$ ). Also let $S(z-$ $x, \lambda)=\sum_{i, j} S^{i j}(z-x, \lambda) E_{i j}, L_{12}^{V}(z, \lambda)=\sum_{i, j} E_{i j} \otimes L_{V}^{i j}(z, \lambda)$ and use the same notation for $L^{W}(z, \lambda)$. Applying (14) to $v_{i} \otimes \zeta_{\mu}$ for some $i$ and $\zeta_{\mu} \in V_{\mu}$ yields

$$
\begin{aligned}
& \sum_{j, k, \nu} S^{k j}\left(z-x, \lambda-\gamma\left(\mu+\omega_{i}-\omega_{j}\right)\right) v_{k} \otimes \varphi_{\nu}(\lambda)\left(L_{V}^{j i}(z, \lambda) \zeta_{\mu}\right) \\
& \quad=\sum_{l, k, \sigma} S^{k l}\left(z-x, \lambda-\gamma\left(\mu+\omega_{i}-\omega_{l}+\sigma\right)\right) v_{k} \otimes L_{W}^{l i}(z, \lambda) \varphi_{\sigma}\left(\lambda-\gamma \omega_{i}\right) \zeta_{\mu}
\end{aligned}
$$

where we used the weight-zero property of $L^{V}(z, \lambda)$ and $L^{W}(z, \lambda)$. Applying $v_{k}^{*}$ to (15) and projecting on the weight space $W_{\mu+\omega_{i}+\xi}$ gives the relation

$$
\begin{aligned}
& \sum_{\substack{\nu, j \\
\nu-\omega_{j}=\xi}} S^{k j}\left(z-x, \lambda-\gamma\left(\mu+\omega_{i}-\omega_{j}\right)\right) \varphi_{\nu}(\lambda)\left(L_{V}^{j i}(z, \lambda) \zeta_{\mu}\right) \\
& \quad=\sum_{\substack{\sigma, l \\
\sigma-\omega_{l}=\xi}} S^{k l}\left(z-x, \lambda-\gamma\left(\mu+\omega_{i}-\omega_{j}+\sigma\right)\right) L_{W}^{l i}(z, \lambda)\left(\varphi_{\sigma}\left(\lambda-\gamma \omega_{i}\right) \zeta_{\mu}\right)
\end{aligned}
$$

for any $i, k, \xi$ and $\zeta_{\mu} \in V_{\mu}$. Now let $A=\left\{\chi \mid \varphi_{\chi}(\lambda) \neq 0\right\}$. Fix some $j$ and let $\beta \in A$ be an extremal weight in the direction $-\omega_{j}$ (i.e $\beta-\omega_{j}+\omega_{k} \notin A$ for $k \neq j$ ). Then (16) for $\xi=\beta-\omega_{j}$ reduces to

$$
\begin{aligned}
& S^{k j}\left(z-x, \lambda-\gamma\left(\mu+\omega_{i}-\omega_{j}\right)\right) \varphi_{\beta}(\lambda)\left(L_{V}^{j i}(z, \lambda) \zeta_{\mu}\right) \\
& \quad=S^{k j}\left(z-x, \lambda-\gamma\left(\mu+\omega_{i}-\omega_{j}+\beta\right)\right) L_{W}^{j i}(z, \lambda) \varphi_{\beta}\left(\lambda-\gamma \omega_{i}\right) \zeta_{\mu}
\end{aligned}
$$

ClaIM. There exists $i \in\{1, \ldots n\}, \mu$ and $\zeta_{\mu} \in V_{\mu}$ such that $\varphi_{\beta}(\lambda)\left(L_{V}^{j i}(z, \lambda) \zeta_{\mu}\right) \neq$ 0 for generic $z$ and $\lambda$.

Proof. Recall the central element $Q \operatorname{det}(z, \lambda) \in \mathcal{E}_{\tau, \frac{\gamma}{2}}\left(\mathfrak{g l}_{n}\right)$. By definition, its action on $V$ is invertible. Expanding $Q \operatorname{det}(z, \lambda)$ along the $j^{\text {th }}$-line, we have $Q \operatorname{det}(z, \lambda)=$ $\sum_{i} L_{V}^{j i}(z, \lambda) P_{i}(z, \lambda)$ for some operators $P_{i}(z, \lambda) \in \operatorname{End}(V)$. In particular,

$$
\sum_{i} \operatorname{Im} L^{j i}(z, \lambda)=V
$$


and the claim follows.

Thus, the ratio $S^{k j}\left(z-x, \lambda-\gamma\left(\mu+\omega_{i}-\omega_{j}+\beta\right)\right) / S^{k j}\left(z-x, \lambda-\gamma\left(\mu+\omega_{i}-\omega_{j}\right)\right)$ is independent of $k$. This is possible only if $\beta \in \sum_{r \neq j} \mathbb{C} E_{r r}^{*}$. Applying this to $j=1, \ldots n$, we see that $A=\{0\}$. Hence $\varphi(\lambda)$ is an $\mathfrak{h}$-module map. But then relation (14) reduces to $\varphi_{2}(\lambda) L_{12}^{V}(z, \lambda)=L_{12}^{W}(z, \lambda) \varphi_{2}\left(\lambda-\gamma h_{1}\right)$, and $\varphi(\lambda)$ is an intertwiner in the category $\mathcal{C}_{F}$. $\square$

Corollary 2. The functor $\mathcal{F}^{c}: \mathcal{C}_{F}^{x} \rightarrow \mathcal{D}_{B}^{x}$ is fully faithful.

6. The image of the vector representation. Let us denote

$$
\tilde{V}_{F}(w)=\left(\mathbb{C}^{n}, \chi(w) R^{F}(w, \lambda)\right)
$$

It is an object of $\mathcal{C}_{F}$ which equals the tensor product of the vector representation $V_{F}(w)$ by the one-dimensional representation $(\mathbb{C}, \chi(z))$.

Proposition 4. For any $x, w, x+c \not \equiv w(\bmod \mathbb{Z}+\tau \mathbb{Z})$, we have $\mathcal{F}_{x}^{c}\left(\tilde{V}_{F}(w)\right) \simeq$ $\mathcal{H}_{x}^{c}\left(V_{B}(w)\right)$.

Proof. By definition, we have

$$
\begin{gathered}
\mathcal{F}_{x}^{c}\left(\tilde{V}_{F}(w)\right)=\left(\mathbb{C}^{n}, \chi(z) S_{1}\left(z-x, \lambda-\gamma h_{2}\right) R^{F}(z-w, \lambda) e^{-\gamma D_{1}} \times S_{1}(z-x-c, \lambda)^{-1}\right), \\
I_{x}^{c} \otimes V_{B}(w)=\left(\mathbb{C}^{n}, \chi(z) S_{1}(z-x, \lambda) e^{-\gamma D_{1}} S_{1}(z-x-c, \lambda) R^{B}(z-w)\right)
\end{gathered}
$$

We claim that the map $\varphi(\lambda)=e^{-\gamma D}\left(S(w-x-c, \lambda)^{-1}\right) e^{\gamma D} \in \operatorname{End}\left(\mathbb{C}^{n}\right)$ is an intertwiner $\mathcal{H}_{x}^{c}\left(V_{B}(w)\right) \simeq I_{x}^{c} \otimes V_{B}(w) \stackrel{\sim}{\rightarrow} \mathcal{F}_{x}^{c}\left(\tilde{V}_{F}(w)\right)$. Indeed, we have

$$
\begin{aligned}
& S_{1}\left(z-x, \lambda-\gamma h_{2}\right) R^{F}(z-w, \lambda) e^{-\gamma D_{1}} S_{1}(z-x-c, \lambda)^{-1}(1 \otimes \varphi(\lambda)) \\
& =e^{-\gamma D_{2}} S_{1}(z-x, \lambda) e^{\gamma D_{2}} R^{F}(z-w, \lambda) e^{-\gamma\left(D_{1}+D_{2}\right)} \\
& \quad S_{1}\left(z-x-c, \lambda+\gamma h_{2}\right)^{-1} S_{2}(w-x-c, \lambda)^{-1} e^{\gamma D_{2}} \\
& =e^{-\gamma D_{2}} S_{1}(z-x, \lambda) e^{-\gamma D_{1}} R^{F}(z-w, \lambda) \\
& \quad S_{1}\left(z-x-c, \lambda+\gamma h_{2}\right)^{-1} S_{2}(w-x-c, \lambda)^{-1} e^{\gamma D_{2}} \\
& =e^{-\gamma D_{2}} S_{1}(z-x, \lambda) e^{-\gamma D_{1}} S_{2}\left(w-x-c, \lambda+\gamma h_{1}\right)^{-1} \\
& \quad S_{1}(z-x-c, \lambda)^{-1} R^{B}(z-w) e^{\gamma D_{2}} \\
& =e^{-\gamma D_{2}} S_{1}(z-x, \lambda) S_{2}(w-x-c, \lambda) e^{-\gamma D_{1}} S_{1}(z-x-c, \lambda)^{-1} R^{B}(z-w) e^{\gamma D_{2}} \\
& =(1 \otimes \varphi(\lambda)) S_{1}(z-x, \lambda) e^{-\gamma D_{1}} S_{1}(z-x-c, \lambda)^{-1} R^{B}(z-w)
\end{aligned}
$$

where we used Lemma 2 and the zero-weight property of $R^{F}(u, \lambda)$.

LEMMA 4. Let $V, V^{\prime} \in \mathcal{O} b\left(\mathcal{C}_{F}\right), W, W^{\prime} \in \mathcal{O} b\left(\mathcal{C}_{B}\right)$ and suppose that $\mathcal{F}_{x}^{c}(V) \simeq$ $\mathcal{H}_{x}^{c}(W)$ and $\mathcal{F}_{x}^{c}\left(V^{\prime}\right) \simeq \mathcal{H}_{x}^{c}\left(W^{\prime}\right)$. Then $\mathcal{F}_{x}^{c}\left(V \otimes V^{\prime}\right) \simeq \mathcal{H}_{x}^{c}\left(W \otimes W^{\prime}\right)$.

Proof. If $\varphi(\lambda): V \rightarrow W$ and $\varphi^{\prime}(\lambda): V^{\prime} \rightarrow W^{\prime}$ are intertwiners then it is easy to check using the methods above that $\varphi_{2}^{\prime}\left(\lambda-\gamma h_{1}\right) \varphi_{1}(\lambda): V \otimes V^{\prime} \rightarrow W \otimes W^{\prime}$ is an intertwiner.

Applying this to tensor products of the vector representations, we obtain

Corollary 3. For any $x \in \mathbb{C}$ and $w_{1}, \ldots, w_{r} \in \mathbb{C} \backslash\{x+c+\mathbb{Z}+\tau \mathbb{Z}\}$, we have

$$
\mathcal{F}_{x}^{c}\left(\tilde{V}_{F}\left(w_{1}\right) \otimes \ldots \tilde{V}_{F}\left(w_{r}\right)\right) \simeq \mathcal{H}_{x}^{c}\left(V_{B}\left(w_{1}\right) \otimes \ldots V_{B}\left(w_{r}\right)\right)
$$


Corollary 4. For any $w_{1}, \ldots, w_{r} \in \mathbb{C}$, we have

$$
\mathcal{F}^{c}\left(\tilde{V}_{F}\left(w_{1}\right) \otimes \ldots \tilde{V}_{F}\left(w_{r}\right)\right) \simeq \mathcal{H}^{c}\left(V_{B}\left(w_{1}\right) \otimes \ldots V_{B}\left(w_{r}\right)\right)
$$

Notice that in this case, we have a canonical intertwiner, given by the formula

$$
\varphi_{1 \ldots r}\left(\lambda, w_{1}, \ldots, w_{r}\right)=\tilde{S}_{r}^{-1}\left(w_{r}-x-c, \lambda-\gamma \sum_{i=1}^{r-1} h_{i}\right) \ldots \tilde{S}_{1}^{-1}\left(w_{1}-x-c, \lambda\right)
$$

where we set $\tilde{S}(z, \lambda)=e^{-\gamma D} S(z, \lambda) e^{\gamma D}$.

7. Equivalence of subcategories. Let us summarize the results of sections 4-8. By proposition 2, we can identify $\mathcal{C}_{B}^{x}$ with a full subcategory $\mathcal{D}_{1}^{x}$ of $\mathcal{D}_{B}^{x}$. By proposition 3 , we can identify $\mathcal{C}_{F}^{x}$ with a full subcategory $\mathcal{D}_{2}^{x}$ of $\mathcal{D}_{B}^{x}$. Moreover, $\mathcal{D}_{1}^{x}$ and $\mathcal{D}_{2}^{x}$ intersect (at least if we replace $\mathcal{D}_{B}^{x}$ by the equivalent category $\widetilde{\mathcal{D}_{B}^{x}}$ whose objects are isomorphism classes of objects of $\mathcal{D}_{B}^{x}$ ), and the intersection contains objects of the form $\mathcal{F}^{c}\left(\bigotimes_{i} \tilde{V}_{F}\left(w_{i}\right)\right) \simeq \mathcal{H}^{c}\left(\bigotimes_{i} V_{B}\left(w_{i}\right)\right)$, where $i=1, \ldots r$ and $w_{i} \in \mathbb{C}$. Hence,

THEOREM The abelian subcategory $\mathcal{V}_{B}^{x}$ of $\mathcal{C}_{B}^{x}$ generated by objects $\bigotimes_{i} V_{B}\left(w_{i}\right)$ for $i=1, \ldots r, r \in \mathbb{N}$ and $w_{i} \in \mathbb{C}$ and the abelian subcategory $\mathcal{V}_{F}^{x}$ of $\mathcal{C}_{F}^{x}$ generated by objects $\bigotimes_{j} \tilde{V}_{F}\left(w_{j}\right)$ for $j=1, \ldots s, s \in \mathbb{N}$ and $w_{j} \in \mathbb{C}$ are equivalent.

Note that for numerical values of $x, \mathcal{F}_{x}^{c}: \mathcal{C}_{F} \rightarrow \mathcal{D}_{B}$ is always fully faithful, and $\mathcal{F}_{x}^{c}\left(\mathcal{C}_{F}\right)$ a full subcategory of $\mathcal{D}_{B}$, but this is not true of $\mathcal{H}_{x}^{c}$, because of the existence of nontrivial finite-dimensional subrepresentations of $I_{x}^{0}$.

\section{REFERENCES}

[1] BAXTER R.J, Eight-vertex model in lattice statistics and one-dimensional anisotropic Heisenberg chain. I, Ann. Phys. 76 (1973) 1-24, II, ibid. 25-47, III, ibid. 48-71.

[2] Belavin A.A, AND Drinfeld V.G, Triangle equation and simple Lie algebras, Soviet Sci. reviews, Sect C 4 93-165 (1984).

[3] Belavin A. A., Dynamical symmetries of integrable quantum systems, Nucl. Phys. B, 180, 189-200 (1981).

[4] Faddeev L., Reshetikhin N., And Takthadjan L., Quantization of Lie groups and Lie algebras, Algebraic Analysis, Vol 1 129-139 Acad. Press (1988).

[5] Felder G., Elliptic quantum groups, preprint hep-th/9412207, to appear in the Proceedings of the ICMP, Paris 1994.

[6] Felder G., AND VARChenko A., On representations of the elliptic quantum group $E_{\tau, \eta}\left(\mathfrak{s l}_{2}\right)$, Comm. Math. Phys. 181 (1996), 746-762.

[7] Felder G., ANd Varchenko A., Algebraic Bethe Ansatz for the elliptic quantum group $E_{\tau, \eta}\left(\mathfrak{s l}_{2}\right)$, preprint q-alg/9605024. Nuclear Physics B 480 (1996), 485-503.

[8] HasegaWA K., Crossing symmetry in elliptic solutions of the Yang-Baxter equation and a new $L$-operator for Belavin's solution, J, Phys. A: Math. Gen. 26 (1993) 3211-3228.

[9] HasegaWA K., L-operator for Belavin's R-matrix acting on the space of theta functions, J. Math. Phys. 35(4) (1994) 6158-6171.

[10] Jimbo M., Miwa T., AND OKAdo M., Local state probabilities of solvable lattice models: an $A_{n}^{(1)}$ family, Nucl. Phys. B300 [FS22] 74-108 (1988).

[11] KRICHEver I., AND ZABRodin A., Spin generalization of the Ruijsenaars-Schneider model, non-abelian $2 D$ Toda chain and representations of the Sklyanin algebra, preprint hepth/9505039.

[12] Reshetikhin N.Y., and Semenov-Tian-Shansky M.A., Central extensions of quantum current groups, Lett. Math. Phys. 19 (1990) 133-142. 